Cite this: DOI: $10.1039 / c 3 n r 03249 c$

\title{
Time resolved study of cell death mechanisms induced by amine-modified polystyrene nanoparticles $\uparrow$
}

\author{
Fengjuan Wang, ${ }^{a}$ Mariana G. Bexiga, ${ }^{\text {abc }}$ Sergio Anguissola, ${ }^{a}$ Patricia Boya, ${ }^{d}$ \\ Jeremy C. Simpson, ${ }^{b}$ Anna Salvati ${ }^{\star a}$ and Kenneth A. Dawson ${ }^{\star a}$ \\ Positively charged polymers and nanoparticles (NPs) can be toxic to cells in various systems. Using human \\ astrocytoma cells, we have previously shown that $50 \mathrm{~nm}$ amine-modified polystyrene NPs damage \\ mitochondria and induce cell death by apoptosis. Here we provide comprehensive details of the cellular \\ events occurring after exposure to the NPs in a time-resolved manner. We demonstrate that the \\ accumulation of NPs in lysosomes plays a central role in the observed cell death, leading to swelling of \\ the lysosomes and release of cathepsins into the cytosol, which ultimately propagates the damage to \\ the mitochondria with subsequent activation of apoptosis. This is accompanied and sustained by other \\ events, such as increasing ROS levels and autophagy. Using various inhibitors, we also show the \\ interplay between apoptosis and autophagy as a response to NP accumulation in lysosomes.
}

Received 24th June 2013

Accepted 19th September 2013

DOI: $10.1039 / c 3 n r 03249 c$

www.rsc.org/nanoscale

\section{Introduction}

Positively charged objects are known to be toxic to cells. Several examples of this come from studies on gene delivery systems, where cationic lipids, polymers and polycations have been often used to condensate and deliver to the cell oppositely charged nucleic acids. ${ }^{1}$ The bare positive charges of several of these gene delivery systems can cause damage to cellular membranes, whereas when covered by DNA they are processed and accumulate intracellularly. ${ }^{2}$ In lysosomes, they can induce lysosomal damage, and in some cases reports have suggested this to be induced via the so called "proton sponge effect". ${ }^{3,4}$ Similarly, bare positively charged NPs have been shown to induce damage to the cell membrane ${ }^{5,6}$ and to have the capacity to reconstruct phospholipid membranes. ${ }^{7}$ Once covered by serum proteins, forming the so called protein corona, ${ }^{8}$ these NPs are trafficked and processed inside the cells and accumulate in the lysosomes. ${ }^{9}$

Several works have reported cell death activated by cationic NPs in various cell types., ${ }^{\mathbf{4} 10}$ Increase in intracellular reactive oxygen species (ROS) and consequent damage to the

${ }^{a}$ Centre for BioNano Interactions and Conway Institute of Biomolecular and Biomedical Research, University College Dublin, Belfield, Dublin 4, Ireland. E-mail: Kenneth.A.Dawson@cbni.ucd.ie; Anna.Salvati@cbni.ucd.ie; Tel: +353 (0)1716 6928

${ }^{b}$ School of Biology \& Environmental Science and Conway Institute of Biomolecular and Biomedical Research, University College Dublin, Belfield, Dublin 4, Ireland ${ }^{c} \mathrm{PhD}$ Programme in Experimental Biology and Biomedicine, Centre for Neuroscience and Cell Biology, University of Coimbra, 3004-517, Coimbra, Portugal

${ }^{d}$ Department of Cellular and Molecular Biology, CIB, CSIC, Ramiro de Maeztu 9, E-28040 Madrid, Spain

$\dagger$ Electronic supplementary information (ESI) available: additional analysis of flow cytometry results, western blots and experiments with cathepsin inhibitors. See DOI: $10.1039 / \mathrm{c} 3$ nr03249c mitochondria have been regarded as crucial steps in the toxicity induced by such positive NPs, which often lead to cell death via apoptosis. ${ }^{4}$ In some cases, damage to lysosomes was observed, however its role has not been fully described. Using human astrocytoma $1321 \mathrm{~N} 1$ cells, we have previously shown that $50 \mathrm{~nm}$ amine-modified polystyrene $\left(\mathrm{NH}_{2}-\mathrm{PS}\right)$ NPs (cationic) induce apoptosis through activation of caspases 3, 7 and 9, followed by the cleavage of PARP-1. ${ }^{10}$ Moreover, we have shown that these NPs, when covered by serum proteins, enter the cells and accumulate in the lysosomes. There, the degradation of this protein layer is accompanied by swelling of the lysosomes and ultimately damage to the lysosomal membrane, with release of the lysosomal content into the cytosol, leading to the observed apoptotic cell death. ${ }^{9}$

In this work, we extend these observations with a detailed time-resolved study of the cellular signals induced by these NPs as they are trafficked inside cells. This allows us to investigate alteration of cellular functions and characteristics, including cell size and granularity (vacuolization), ROS generation, lysosomal integrity and morphology, mitochondrial membrane potential, intracellular and mitochondrial calcium levels, exposure of phosphatidylserine and plasma membrane integrity.

We also show activation of autophagy upon exposure to the NPs and study its role in the observed cell death. Autophagy is a constitutive process in which lysosomes play a crucial role. During autophagy, a portion of the cytoplasm or old and damaged organelles is engulfed by double or multi membrane structures (autophagosomes), which later fuse with lysosomes and are digested by lysosomal enzymes. Lysosomal dysfunction has been shown to affect the autophagic flux. ${ }^{11}$ Here we examine the formation of autophagosomes and their intracellular localization, in order to connect this process to the lysosomal damage induced upon accumulation of $\mathrm{NH}_{2}$-PS NPs. 
Moreover we propose possible links between the observed apoptosis and autophagy: it is the balance between these two processes, which cells can utilize as responses to cellular stress, to decide the final cellular fate. ${ }^{12}$ It has been previously reported that lysosomotropic agents can cause cytoplasmic vacuolization and cell death that involves hallmarks of both apoptosis and autophagy. ${ }^{\mathbf{1 3 , 1 4}}$ By using inhibitors for these two pathways to modulate the response of the cells, we can further clarify how apoptosis and autophagy are interconnected in the cell death induced by $\mathrm{NH}_{2}-\mathrm{PS}$ NPs. Finally, we use cathepsin inhibitors to clarify the role of the release of lysosomal content into the cytosol in the observed cell death.

Overall, thanks to population discrimination analysis of the results obtained from individual cells by flow cytometry, and by combining the overall sequence of the cellular events detected in this system, we propose a more comprehensive and sequential description of the damage induced by $\mathrm{NH}_{2}-\mathrm{PS}$ NPs. This allows us to understand the relation between the signals activated upon exposure to NPs and NP location inside the cells and ultimately the mechanisms of cell death. This time-resolved approach suggests that most of the observed signals can be explained with the damage at lysosomal level and that this event is upstream to the other cell death events, including the damage to mitochondria and activation of caspases, thus playing a central role in the impact of these NPs on cells.

\section{Experimental details}

\subsection{Cell culture}

Human brain astrocytoma $1321 \mathrm{~N} 1$ cells were obtained from the European Collection of Cell Cultures (ECACC). Cells were routinely cultured in Dulbecco's Modified Eagle's Medium Glutamax (DMEM) (Life Technologies) supplemented with 10\% heat-inactivated foetal bovine serum (FBS) (Life Technologies) and 50 units $\mathrm{ml}^{-1}$ of penicillin and $50 \mu \mathrm{g} \mathrm{ml} \mathrm{m}^{-1}$ of streptomycin (complete cell culture medium, cDMEM) in a $37{ }^{\circ} \mathrm{C}$ incubator with $95 \%$ air/5\% $\mathrm{CO}_{2}$ atmosphere. Serum heat inactivation was performed by incubating the serum at $56{ }^{\circ} \mathrm{C}$ for 30 minutes.

\subsection{Flow cytometric measurement of cellular functions}

$1321 \mathrm{~N} 1$ cells were grown to $70-80 \%$ confluency and exposed to $50 \mu \mathrm{g} \mathrm{ml}^{-1}$ amine-modified $\mathrm{NH}_{2}-\mathrm{PS}$ NPs (fluorescence: $\lambda_{\text {ex }} \sim 360$ $\mathrm{nm} ; \lambda_{\mathrm{em}} \sim 420 \mathrm{~nm}, 50 \mathrm{~nm}$, Sigma Aldrich) in cDMEM for the indicated times $(1,3,6,8$ and $24 \mathrm{~h})$. To expose cells to NPs, 300000 cells were seeded into $3 \mathrm{~cm}$ diameter plates and $24 \mathrm{~h}$ after seeding, the medium was replaced by $1 \mathrm{ml}$ freshly prepared $50 \mu \mathrm{g} \mathrm{ml} \mathrm{m}^{-1}$ amine-modified $\mathrm{NH}_{2}$-PS NP dispersion in cDMEM. NP characterization in PBS and CDMEM was published elsewhere. ${ }^{9,10}$ Cells were harvested with trypsin and stained with various fluorescent markers in cDMEM for $20 \mathrm{~min}$ at $37^{\circ} \mathrm{C}$ in darkness, unless stated otherwise. Cell fluorescence intensity was measured immediately after staining using a Cyan ADP flow cytometer (Beckman Coulter). For the assessment of lysosomal stability, cells were stained with $50 \mathrm{nM}$ LysoTracker Red (Life Technologies), an acidotropic dye that accumulates in acidic compartments; a $30 \mathrm{~mW} 488 \mathrm{~nm}$ argon-ion laser was used to excite the dye and its emission was collected using a $613 \pm 20 \mathrm{~nm}$ band pass filter (red channel, FL2). For the measurement of intracellular ROS levels, cells were stained with $2.5 \mu \mathrm{M}$ CM- $\mathrm{H}_{2}$ DCFDA (Life Technologies) which becomes fluorescent when oxidized by intracellular ROS; a $30 \mathrm{~mW} 488 \mathrm{~nm}$ laser was used to excite the dye and its emission was collected using a $530 \pm 40 \mathrm{~nm}$ band pass filter (green channel, FL1). To evaluate the mitochondrial membrane potential, cells were stained with 1 $\mu \mathrm{g} \mathrm{ml} \mathrm{JC}^{-1}$ (Molecular Probes), a cationic dye that accumulates in mitochondria depending on their transmembrane potential. A $30 \mathrm{~mW} 488 \mathrm{~nm}$ laser was used to excite the dye and its emission was collected in both FL 1 and FL2 channels; the increase in green fluorescence intensities in the FL1 channel was used as a measurement of loss of mitochondrial membrane potential (see Fig. S5C $\uparrow$ for details). To examine plasma membrane integrity and phosphatidylserine exposure (as a sign of apoptosis), cells were stained with $5 \mu \mathrm{l}$ of annexin V-FITC (Calbiochem) in $195 \mu \mathrm{l}$ binding buffer (Calbiochem) for $10 \mathrm{~min}$ at room temperature and propidium iodide (PI) (Sigma Aldrich) was added to reach a concentration of $2 \mu \mathrm{g} \mathrm{ml} \mathrm{m}^{-1}$ just before the measurement; a 30 $\mathrm{mW} 488 \mathrm{~nm}$ laser was used to excite both dyes; the FL1 channel was used to collect the emission of annexin V-FITC, and the FL2 channel was used to collect the emission of PI. Quantitative analysis of flow cytometry data was carried out using Summit software (DAKO). Gates were set to discriminate cell debris and cell doublets from the analysis according to their forward and side scattering. A minimum of 15000 cells were acquired for each sample. The obtained fluorescence intensity distributions and double scatter plots of cell side scattering versus forward scattering are shown. Additional graphs in ESI $\dagger$ show the mean fluorescence intensities of the distributions averaged over three replicates and error bars are their standard deviation. Results are representative of three independent experiments, each performed with three replicates.

\subsection{Western blotting of LC3}

Cells were treated as previously described and whole cell extracts were prepared using RIPA lysis buffer (Thermo Fisher Scientific) supplemented with a protease inhibitor cocktail (Roche). Protein concentration was determined using the BCA assay (Thermo Fisher Scientific) following the manufacturer's instructions. $15 \mu \mathrm{g}$ of total proteins were separated by SDSPAGE followed by protein transfer to a PVDF membrane which was probed with a rabbit polyclonal LC3 antibody (Cell Signaling Technology) or a rabbit polyclonal $\beta$-actin antibody (Abcam), followed by staining with a secondary goat anti rabbit HRP antibody. Proteins were revealed using ECL reagent (Thermo Fisher Scientific). Densitometry was performed with ImageJ software (National Institutes of Health).

\subsection{Immunostaining and confocal fluorescence microscopy}

In order to prepare samples for imaging, collagen coated coverslips were placed on the bottom of a $3 \mathrm{~cm}$ diameter plate and cells seeded as described above prior to exposure to $1 \mathrm{ml}$ $50 \mu \mathrm{g} \mathrm{ml} \mathrm{m}^{-1} \mathrm{NH}_{2}$-PS NP dispersion in cDMEM for indicated times ( $1 \mathrm{~h}, 3 \mathrm{~h}, 6 \mathrm{~h}$ and $8 \mathrm{~h})$. Cells were fixed with methanol for 
4 min at $-20{ }^{\circ} \mathrm{C}$. Mouse monoclonal antibodies for LC3 (Nanotools) or LAMP-1 (Abcam) were used to mark autophagosomes and lysosomes, respectively, followed by staining with a goat anti-mouse Alexa 488 secondary antibody (Life Technologies). Images were acquired on a FluoView FV1000 confocal microscope (Olympus, Hamburg, Germany) equipped with a $60 \times / 1.35 \mathrm{NA}$ objective. $\mathrm{NH}_{2}$-PS NPs were excited with a $405 \mathrm{~nm}$ laser; Alexa 488 secondary antibody was excited with a $488 \mathrm{~nm}$ laser.

\subsection{Treatment with various inhibitors of cellular pathways}

Cells were pre-treated with either $20 \mu \mathrm{M}$ pan- caspase inhibitor zVAD-FMK (zVAD, Calbiochem) or $2 \mathrm{mM}$ autophagy inhibitor 3methyladenine (3 MA, Sigma Aldrich) for $1 \mathrm{~h}$, or $50 \mu \mathrm{M}$ pepstatin A (Pep A) or $30 \mu \mathrm{M}$ E64d (Sigma Aldrich) for $16 \mathrm{~h}$ prior to exposure to the $\mathrm{NH}_{2}$-PS NPs in the presence of the same inhibitors.

\subsection{Measurement of ATP content and caspase 3/7 activity}

For ATP content and caspase activity measurements, 12000 cells were seeded into 96 well plates and exposed to $50 \mu \mathrm{l} 50 \mu \mathrm{g}$ $\mathrm{ml}^{-1} \mathrm{NH}_{2}$-PS NP dispersion in cDMEM for $24 \mathrm{~h}$ with or without the different inhibitors as specified above. Measurement of ATP content was carried out using the CellTiter-Glo kit (Promega), and caspase 3/7 activity was determined using Caspase 3/7-Glo kit (Promega) according to manufacturer's instructions. Relative luminescent units (RLU) were detected with Varioskan Flash plate reader (Thermo Scientific). The results have been averaged between three replicates and all results have been normalized to the values obtained for untreated control cells in the same conditions. Error bars represent the standard deviations between the 3 replicates. Each experiment was performed at least three times.

\section{Results and discussion}

\subsection{Lysosomal alterations precede apoptosis}

3.1.1. Alteration of cellular size and granularity. 1321N1 cells were exposed to $\mathrm{NH}_{2}$-PS NPs for increasing periods of time and analyzed by flow cytometry. These time resolved experiments allowed us to analyze changes in the forward scatter (FS, connected to cell size) and side scatter (SS, related to cell granularity) of the cells and showed that after exposure to the $\mathrm{NH}_{2}$-PS NPs, two distinct populations could be recognized and easily distinguished (colored in blue and red in the FS-SS plots in Fig. 1A). The majority of the population (in blue) showed unchanged FS (Fig. S2C $\dagger$ ) and increasing SS (Fig. S2D $\dagger$ ) with increasing exposure time to the NPs, when compared to untreated control cells $(0 \mathrm{~h})$. This is likely to result from an elevated degree of vacuolization of the cells, ${ }^{\mathbf{1 4}}$ which is consistent with light microscopy data (see phase contrast images in Fig. S1 $\dagger$ ) and which has previously been observed by electron microscopy (EM) imaging..$^{10}$ The early and continuous increase of SS upon exposure to the NPs suggests that the vacuolization is one of the earlier cellular responses after treatment with these NPs. The second population (in red) included cells with very low FS, which clearly correspond to dying cells, in this case late apoptotic cells. The percentage of cells in this second population increased with increasing exposure time (Fig. S2B $\dagger$ ). Already at early exposure times (3-6 h), $10 \%$ of the cells belonged to this sub-population, suggesting that within the macroscopic cell populations, NPs entered and affected individual cells at different times and to a different extent. (This is also visible in the phase contrast images in Fig. S1 $\uparrow$ where already after $6 \mathrm{~h}$ of exposure, some cells were spherical, indicative of cell death, while most cells still seemed unaffected). For this reason, we distinguished these two different populations in the following analysis and assessed their cellular functions and signals separately, using the different cell FS to distinguish them (the same gates and color codes as shown in Fig. 1A for the FS-SS plots are used to facilitate the observation of the results).

3.1.2. Increase of volume of the acidic compartments, and loss of lysosomal membrane integrity. One possible explanation of the increase in SS observed upon treatment with the $\mathrm{NH}_{2}$-PS NPs can be an increase in the volume of acidic compartments in the cells. To analyze this, we used the acidotropic dye LysoTracker Red which accumulates and stains the acidic compartments of the cells, mainly lysosomes. As shown in Fig. 1B and further analyzed in Fig. S2 $\uparrow$ (data reproduced from Wang et al. ${ }^{9}$ to allow comparison), an increase of LysoTracker Red fluorescence intensity in the main cell population (blue) was detected after 3-6 h exposure (Fig. S2E $\dagger$ ). This effect correlated with the enlargement observed using LAMP-1 staining (a lysosomal marker) in the lysosomes where the NPs accumulated (Fig. 2A). Together these results indicate an increased volume of the acidic compartments.,15 Similar effects have been reported with other cationic NPs and lysosomotropic reagents. ${ }^{15,16}$ This effect has been explained in some cases with the so-called "proton sponge theory": some polycations can become protonated in the lysosomes, thereby leading to an alkalization of these organelles, to which the cell reacts by actively pumping more protons into their lumen; to maintain the osmotic and electrochemical gradient, this proton flux is accompanied by a water and ion flux, which ultimately causes swelling of the lysosomes and other related lysosomal damage. ${ }^{4}$ However not all cationic species can induce such effects and we have previously reported similar swelling also for non protonable cationic nanoparticles, ${ }^{9}$ thus suggesting that proton sponge effects, if present, are not the only cause of the observed lysosomal alterations, and that other mechanisms may be involved.

At 6-8 $\mathrm{h}$ after exposure to NPs, a second population with lower LysoTracker Red staining could be identified, constituting approximately $20 \%$ of the whole cell population. The loss of LysoTracker Red fluorescence can be explained by loss of lysosomal integrity, ${ }^{\mathbf{1 7}}$ which can lead to release of lysosomal proteases such as cathepsins $\mathrm{B}, \mathrm{D}$ and $\mathrm{L}$ into the cytosol. ${ }^{18}$ These proteases can indeed be detected in the cytosol after $8 \mathrm{~h}$ exposure to the NPs, ${ }^{9}$ as shown in Fig. S3. $\dagger$ These more severely damaged lysosomes could derive from the enlarged lysosomes observed by immunostaining, as large lysosomes have been suggested to be more susceptible to rupture. ${ }^{19}$ 

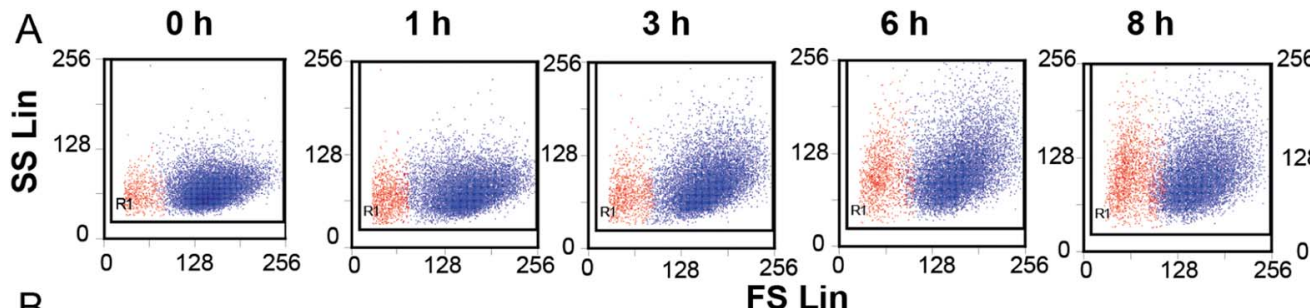

$24 \mathrm{~h}$
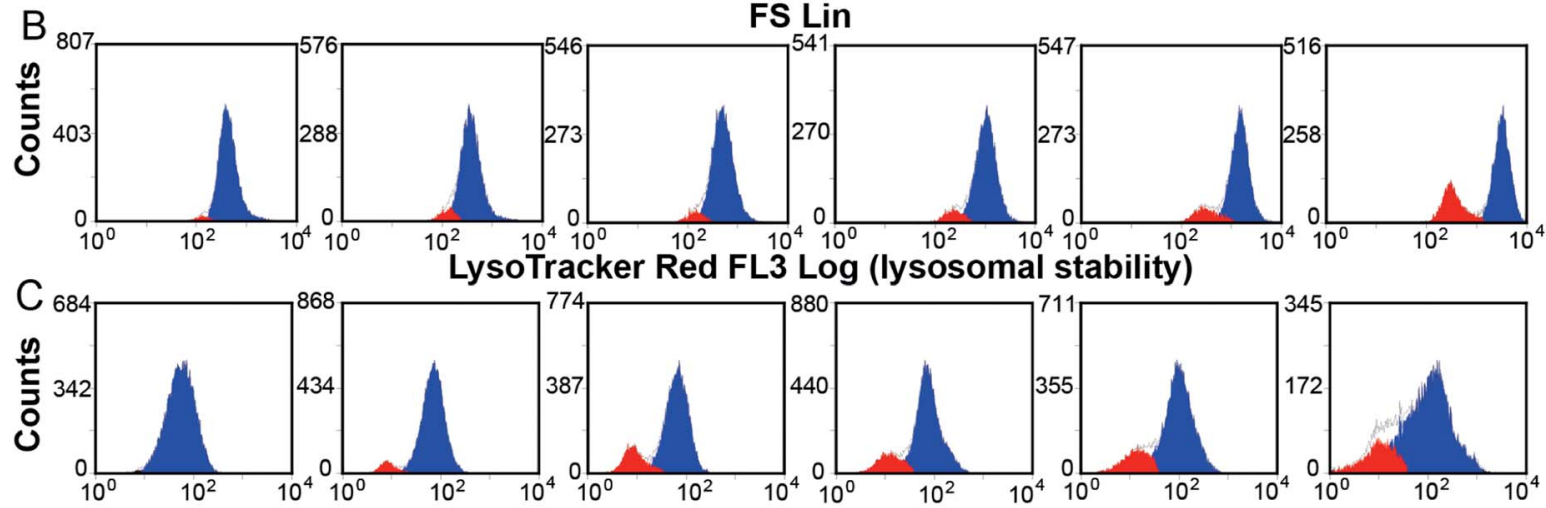

CM-H DCFDA FL 1 Log (ROS)
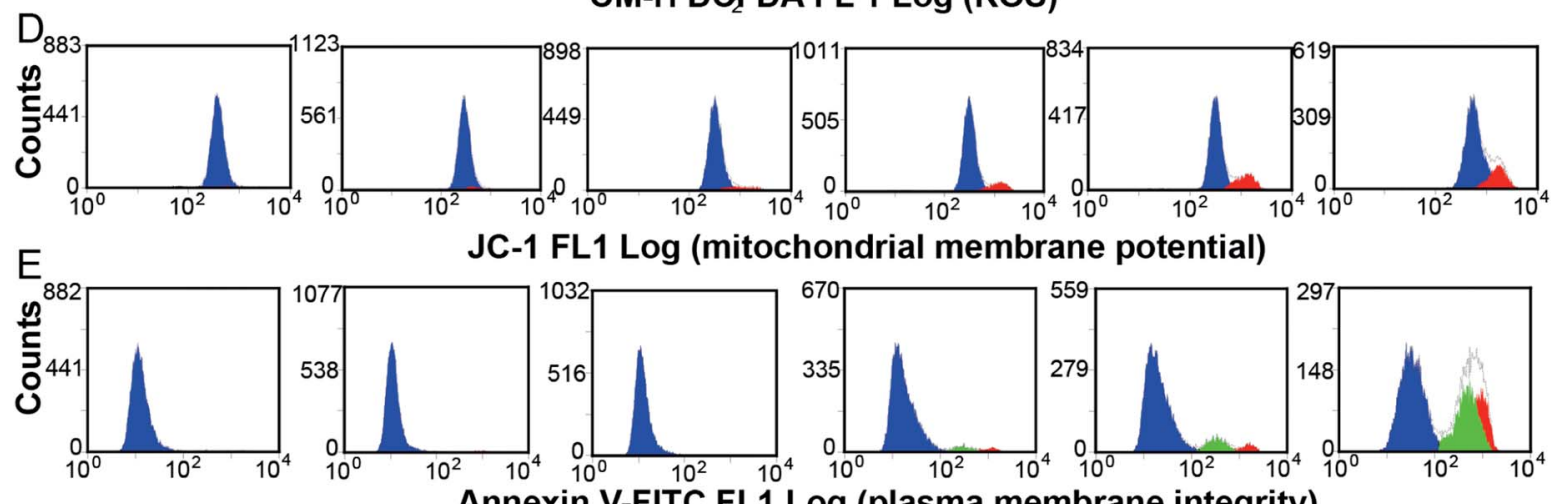

Fig. 1 Time-resolved impact of $\mathrm{NH}_{2}-\mathrm{PS}$ NPs on cellular functions by flow cytometry. $1321 \mathrm{~N} 1$ cells were treated with $50 \mu \mathrm{g} \mathrm{ml} \mathrm{l}^{-1} \mathrm{NH}_{2}-\mathrm{PS} \mathrm{NPs}$ for increasing time (1 h, 3 h, $6 \mathrm{~h}, 8 \mathrm{~h}, 24 \mathrm{~h}$ ) and stained with different fluorescent dyes to measure the corresponding cellular function by flow cytometry. (A) Dot plots of side scatter (SS Lin) versus forward scatter (FS Lin) related to the size and granularity of cells. (B) Distribution of LysoTracker Red fluorescence intensity (Logarithmic scale), showing that after treatment with NPs, a shift of the main cell population (blue) to higher fluorescence intensities was observed, followed by the formation of a second population of cells (red) with lower LysoTracker Red intensity, due to loss of lysosomal integrity. (Data reproduced from Wang et al. ${ }^{9}$ to allow comparison). (C) Distribution of CM- ${ }_{2}$ DCFDA fluorescence intensity (Logarithmic scale), showing increasing ROS generation in the main cell population (blue) at increasing exposure times. Cells in late stages of cell death (red) are not stained by the dye. (D) Distribution of JC-1 fluorescence intensity (Logarithmic scale), showing increasing percentage of cells with loss of mitochondrial membrane potential (red) at increasing exposure time. (E) Distribution of annexin V-FITC fluorescence intensity (Logarithmic scale), showing exposure of phosphatidylserine at increasing exposure time (early apoptotic cells, green), followed by cell death (late apoptotic and necrotic cells, red). See ESI $\dagger$ and main text for more details on the gates applied.

3.1.3. Increase in intracellular reactive oxygen species (ROS). Lysosomal damage can lead to ROS generation, creating a feedback loop which can further amplify the damage. ${ }^{17}$ Here we measured intracellular ROS generation after exposure to $\mathrm{NH}_{2}$-PS using CM- $\mathrm{H}_{2}$ DCFDA staining followed by flow cytometry analysis (Fig. 1C). Two populations could again be clearly observed by plotting the cell FS against the CM- $\mathrm{H}_{2}$ DCFDA fluorescence (as shown in Fig. S4 $\dagger$ ). After 3-6 h exposure, the main population (blue) showed increased intracellular ROS levels at increasing exposure times, with an approximate 2 fold increase in respect to the untreated cells (Fig. S4C $\dagger$ ). ROS generation can be directly related to the capacity of certain NPs, such as metal oxide NPs, to generate ROS, ${ }^{20}$ however this is not expected in the case of polystyrene. Moreover, since the NP surface is covered by the protein corona until accumulation in the lysosomes, ${ }^{9}$ we suspect that in this case the ROS generation, at least partially, is a consequence of the release of lysosomal content into the cytosol rather than a direct production on the NP surface. The population of cells with lower FS (red) had a low $\mathrm{CM}-\mathrm{H}_{2}$ DCFDA intensity (ESI, Fig. S4C $\dagger$ ). These, as explained before, are cells in late stage cell death, which probably are not stained by the fluorescent dye.

3.1.4. Loss of mitochondrial membrane potential and exposure of phosphatidylserine. The high production of ROS in response to $\mathrm{NH}_{2}-\mathrm{PS} \mathrm{NP}$ accumulation suggested that mitochondrial damage could also be induced. Most cell death mechanisms can converge at the level of the mitochondria and loss of mitochondrial membrane potential $(\Delta \Psi \mathrm{m})$ is usually 
A

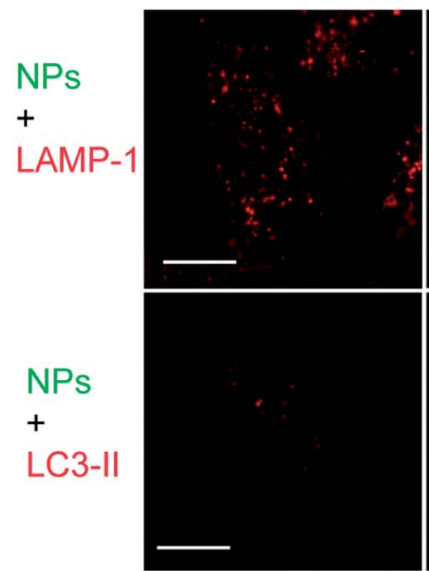

B

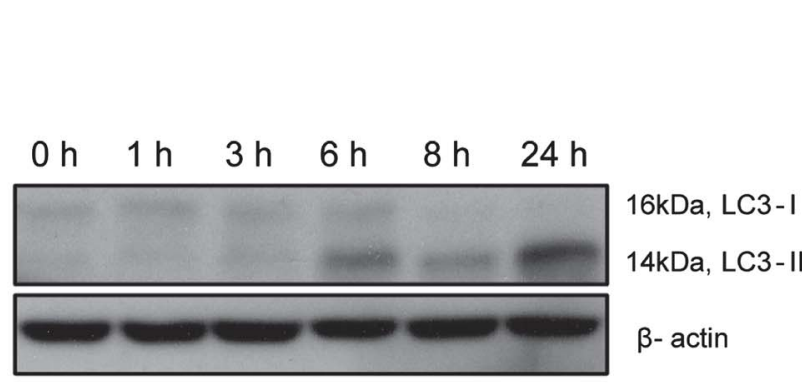

$1 \mathrm{~h}$

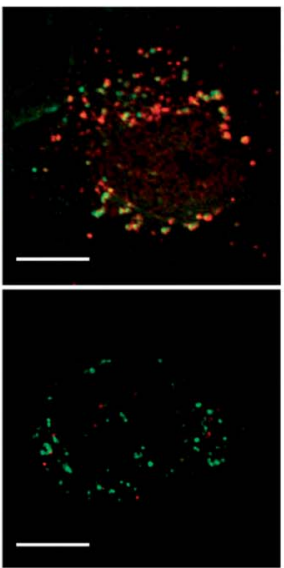

$3 \mathrm{~h}$
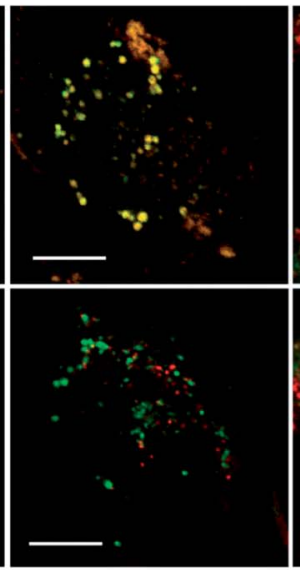

C
$6 \mathrm{~h}$
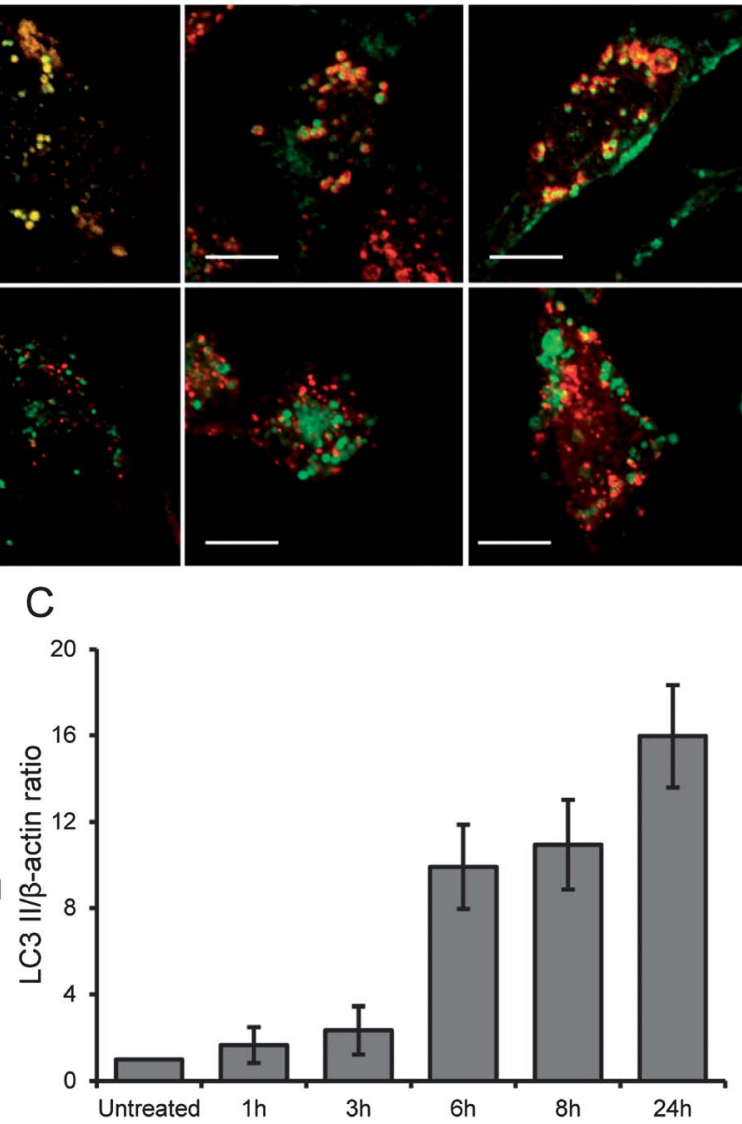

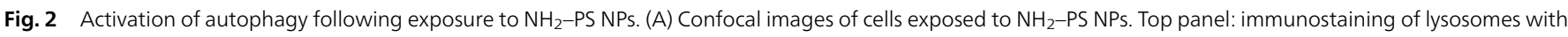

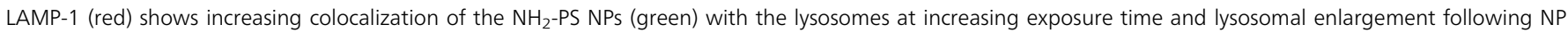

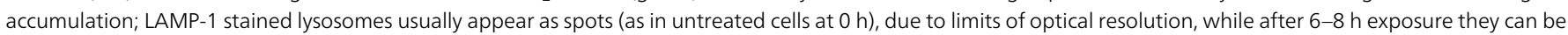

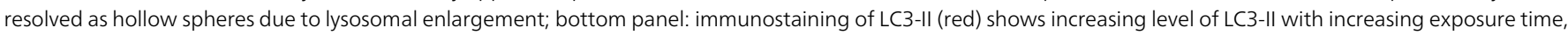

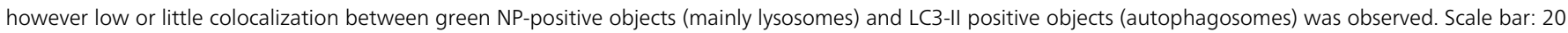

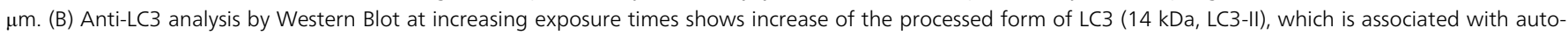

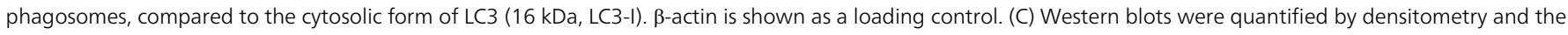

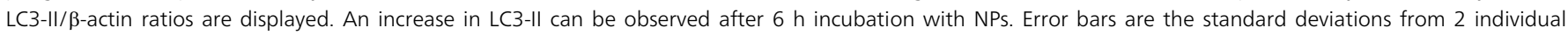
experiments.

regarded as the "point-of-no-return" in cell death. ${ }^{21,22}$ In order to monitor changes in the mitochondrial membrane potential, we stained the cells using JC-1, a cationic dye that accumulates in mitochondria depending on their transmembrane potential. Staurosporine (STS) was used as a positive control known to induce loss of mitochondrial membrane potential (see Fig. S5† for details). With JC-1 staining, we observed that $20 \%$ of cells showed loss of $\Delta \Psi \mathrm{m}$ after $8 \mathrm{~h}$ of exposure to the $50 \mu \mathrm{g} \mathrm{ml}$ $\mathrm{NH}_{2}$-PS NPs (Fig. 1D, and full kinetics in Fig. S5B $\dagger$ ) and this percentage increased at increasing exposure times.

Overall the time resolved analysis indicated that loss of mitochondrial membrane potential was detected only after $8 \mathrm{~h}$, thus later than lysosomal alterations which occurred already after 3-6 $\mathrm{h}$ exposure. Moreover, the cells with loss of mitochondrial potential could be easily distinguished in FS-SS double scatter plots due to their lower FS, as also observed for cells with loss of Lysotracker staining, suggesting that they were the same cells. This all suggested that the lysosomal damage and alterations may be upstream to the observed mitochondrial damage. Many cathepsins have been shown to activate proapoptotic proteins, which can in turn lead to mitochondrial membrane permeabilization and downstream events such as activation of caspase $3 / 7$ and cleavage of PARP-1. ${ }^{23,24}$ Indeed caspase activation and PARP-1 cleavage have been observed upon exposure to these NPs in the same time scale. ${ }^{9,10}$

Exposure of phosphatidylserine at the cell surface is a caspase dependent process that constitutes a recognition signal for phagocytic cells to engulf apoptotic cells. ${ }^{22}$ With annexin V-FITC and PI double staining, we could detect phosphatidylserine exposure (Annexin V positive/PI negative cells, marked in green in Fig. $1 \mathrm{E}$ and $\mathrm{S} 6 \mathrm{~A} \dagger$ ) after $6-8 \mathrm{~h}$ exposure to $\mathrm{NH}_{2}-\mathrm{PS}$ NPs, thus in agreement with the detection of caspase activation. This early apoptotic event is usually followed at later times by plasma membrane permeabilization (Annexin V/PI double positive cells, marked in red in Fig. 1E and Fig. S6A $\dagger$ ), indication of late stage of cell death. Overall, annexin V/PI staining showed a 
steady increase in the percentage of early apoptotic and late apoptotic/necrotic cells at increasing exposure times, which was mirrored by a steady decrease in the fraction of healthy cells (double negative staining).

In summary, $\mathrm{NH}_{2}-\mathrm{PS}$ NPs induced an increase in cellular vacuolization and the volume of acidic compartments within 3$6 \mathrm{~h}$ of exposure; after 6-8 h, loss of LysoTracker Red staining and release of cathepsins (B, D and L) were detected, followed by loss of $\Delta \Psi \mathrm{m}$ and other apoptotic hallmarks, such as caspase activation, exposure of phosphatidylserine on the cell surface and PARP-1 cleavage. Altogether this series of events suggests lysosomal damage can be an upstream proapoptotic event. Once the lysosomes are broken and their contents (such as cathepsins $\mathrm{B}, \mathrm{D}$ and $\mathrm{L}$ ) released, damage to mitochondria follows, and is amplified and sustained by ROS generation. The damage to mitochondria and lysosomes is also accompanied by increased cytosolic calcium (Fig. S7 $\dagger$ ) and mitochondrial calcium (Fig. S8 $\dagger$ ) levels. The increase in mitochondrial calcium can be a consequence of intracellular calcium overload and can also contribute to mitochondrial damage and apoptosis. ${ }^{21}$

\subsection{Accumulation of autophagosomes}

3.2.1. Increase of autophagosomes after treatment with $\mathbf{N H}_{2}$-PS NPs. Lysosomal damage can have consequences on other cellular pathways, such as, for instance, autophagy. ${ }^{\mathbf{1 1 , 2 5}}$ We have previously reported that a large degree of vacuolization could be observed after treatment with $\mathrm{NH}_{2}$-PS NPs, suggesting that autophagy could be involved, ${ }^{\mathbf{1 0}}$ possibly explaining the observed increase in SS detected by flow cytometry (Fig. 1A). Induction of autophagy leads to formation of autophagosomes which recruit LC3 II proteins (alternatively known as MAP1LC3A II, microtubule associated protein 1 light chain 3 alpha II) to their membranes. In order to further investigate this, we quantified by Western blotting the cellular level of LC3 II. $^{26}$ Autophagy is a highly dynamic process which can be hard to reproduce quantitatively. However, results clearly showed that at 6-8 $\mathrm{h}$ after exposure to the $\mathrm{NH}_{2}-\mathrm{PS}$ NPs a significant increase of LC3 II levels could be observed (Fig. 2B and C). This could be due to either induction of autophagy or a defect in autophagosomal clearance in the lysosomes. Recent studies have in fact pointed out that an increase of LC3 II levels only indicates the accumulation of autophagosomes, while the autophagic flux depends on the fusion of the LC3 II positive autophagosomes with the lysosomes and following degradation of LC3 II and other contents engulfed by autophagosomes in the lysosomes. Impairment of this flux can also lead to an increase in LC3 II positive membranes which accumulate inside the cell without being degraded inside lysosomes. ${ }^{27,28}$ Further studies are required to fully elucidate this.

3.2.2. Relation between NP localization, lysosomes and autophagosomes. Analysis of the subcellular localization of the $\mathrm{NH}_{2}$-PS NPs, lysosomes and autophagosomes can provide additional information on the relation between lysosomal damage and autophagy. Using immunostaining and confocal microscopy imaging, as previously reported, NPs were found to co-localize with LAMP-1 positive objects (lysosomes) after 1-3 h incubation, while lysosomal enlargement was observed after 3-6 h (Fig. 2A top panel), consistent with the increase of LysoTracker Red staining discussed above (Fig. 1B). Immunostaining of the autophagosomes (LC3 antibody) showed that after 3-6 h incubation with $\mathrm{NH}_{2}-\mathrm{PS}$ NPs, LC3 II (which can be distinguished as punctate structures by LC3 staining) increased in comparison to untreated control cells (Fig. 2A bottom panel). This result correlates with the cleavage of LC3 II observed by Western Blot analysis (Fig. 2B and C), suggesting generation of autophagosomes upon exposure to the NPs. Intriguingly, although NPs colocalized with LAMP-1 positive objects (lysosomes), large part of the LC3 II positive objects (autophagosomes) did not colocalize with NPs, even after $8 \mathrm{~h}$. This may suggest that the autophagic flux to the lysosomes could be impaired. ${ }^{\mathbf{1 1 , 2 9}}$ However, more studies are required to fully investigate this.

\subsection{Interplay between the different cell death pathways}

In order to better understand the interplay between the different cell death pathways activated upon exposure to the $\mathrm{NH}_{2}$-PS NPs, we have compared cell death levels after inhibition of caspases, autophagy or cathepsins.

3.3.1. Inhibition of caspases does not protect cells from the damage induced by NH2-PS NPs. We have previously reported that $\mathrm{NH}_{2}$-PS NPs induce apoptosis by activation of caspase 3/7 and $9 .{ }^{10}$ Caspase- dependent cell death can often be rescued or delayed, at least in part, when the activity of caspases is inhibited by $\mathrm{z}$-VAD, a broad spectrum caspase inhibitor. ${ }^{30}$ In order to investigate whether the cell death induced by the $\mathrm{NH}_{2}^{-}$ PS NPs was dependent on caspases, cells were pre-incubated with zVAD and ATP levels were measured to assess the level of cell death. Fig. 3A shows that after $24 \mathrm{~h}$ incubation with $\mathrm{NH}_{2}-\mathrm{PS}$ NPs, cells pre-treated with zVAD had low ATP levels as cells exposed to the only NPs (without zVAD). This indicates that inhibition of caspases did not protect cells from the damage induced by the NPs. This finding may be not surprising, considering that strong damage to the lysosomes with release of lysosomal content in the cytosol can propagate the cell damage to mitochondria, independently from caspase activity. ${ }^{23}$

\subsubsection{Inhibition of autophagy sensitizes cells to $\mathrm{NH}_{2}-\mathrm{PS}$} NPs- induced cell death. Autophagy can be activated by cells as a pro-survival mechanism in response to cellular stress, and to sustain energy production via alternative routes, but it can also be engaged in a cell death program, depending on the stimuli and the cell type. ${ }^{12,31}$ We have shown that the cell death induced by $\mathrm{NH}_{2}$-PS NPs is accompanied by increased LC3 II structures (Fig. 2). In order to investigate the effect of autophagy on the cell death levels, we used 3 MA to block autophagy at the initiation step by inhibition of phosphatidylinositol 3-kinase, an enzyme required for the sequestration of autophagy. ${ }^{27}$ ATP levels were measured after $24 \mathrm{~h}$ of exposure to the $\mathrm{NH}_{2}-\mathrm{PS}$ NPs with or without $3 \mathrm{MA}$ as an indicator of cell viability. The results showed that in the presence of 3 MA the ATP levels after exposure to NPs were lower than in cells exposed to NPs only (Fig. 3A); similarly caspase $3 / 7$ activation was higher when 3 MA was present (Fig. 3B). These results indicated that the combination 
A
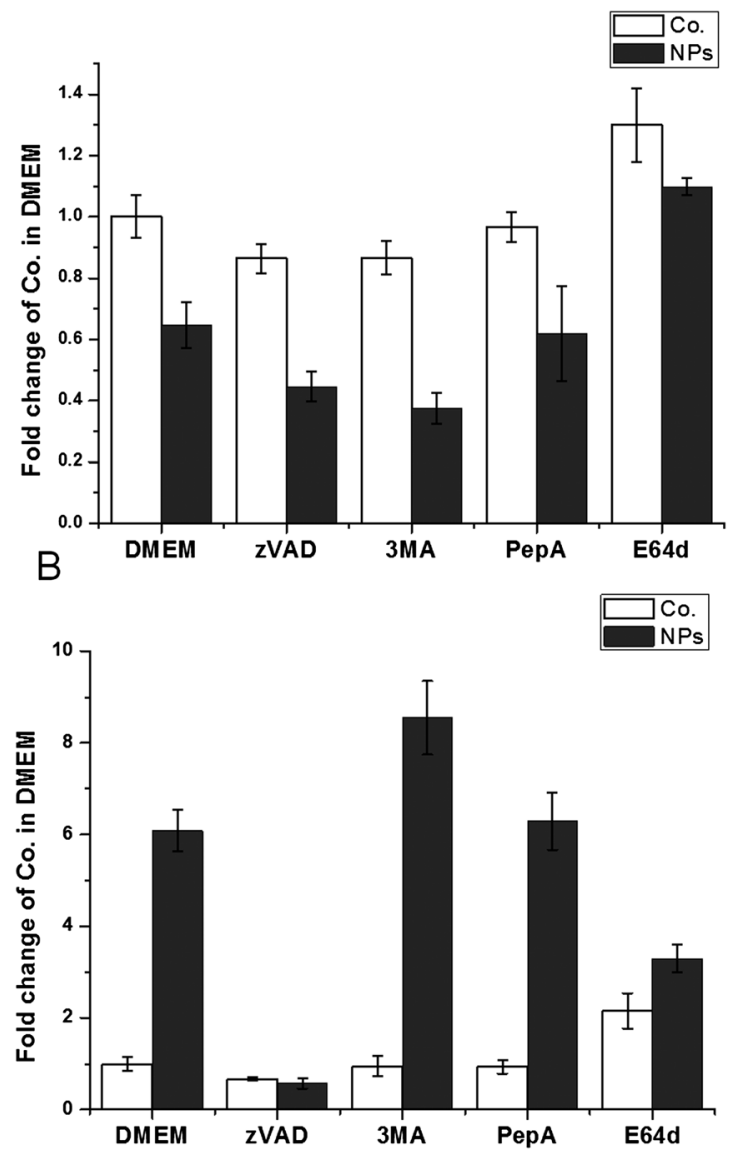

Fig. 3 Study of cell death pathways with inhibitors. Cells were treated with 50 $\mu \mathrm{g} \mathrm{ml^{-1 }}$ NPs for $24 \mathrm{~h}$ with or without pan-caspase inhibitor ZVAD $(20 \mu \mathrm{M})$, cathepsin B/L inhibitor E64d $(30 \mu \mathrm{M})$, cathepsin D inhibitor pepstatin $\mathrm{A}(50 \mu \mathrm{M})$ or autophagy inhibitor 3-methyladenine (2 mM). (A) ATP content was used to assess the level of cell death and demonstrated that inhibition of cathepsin $D, B / L$ can delay cell death, but not zVAD nor 3 MA. (B) Caspase 3/7 activity levels were used to assess the level of apoptosis and showed that inhibition of cathepsin $B / L$ with E64d decreased the level or caspase 3/7 activation, but not the other inhibition treatments. The open bars show the results obtained on control cells not exposed to the NPs (Co.) and solid black bars show the results for NP- treated cells (NPs). All results for NP-treated cells were normalized for the values obtained on untreated control cells (not exposed to NPs) pre-incubated with individual inhibitors. Results are the mean of 3 experiments, each with 3 replicates, and the error bars are the standard deviation.

of $\mathrm{NH}_{2}$-PS NPs and 3 MA had a synergistic lethal effect. Thus, the activation of autophagy might play a protective role at early exposure times (in the first $6 \mathrm{~h}$ ) in order to help to maintain high ATP levels in response to the damage induced by the NPs. ${ }^{10}$

3.3.3. Role of cytosolic release of lysosomal content, such as cathepsins $B, D$ and $L$, in cell death. As shown above, the loss of LysoTracker Red staining after 6-8 h incubation with NPs (Fig. 1) is followed by the release of lysosomal content, such as cathepsins, into the cytosol (Fig. S3†). Cathepsins B, D and L are the most abundant cathepsins which normally reside inside lysosomes. $^{32}$ The release of cathepsins B, D and L from lysosomes into the cytosol can cause digestion of vital proteins and the activation of additional hydrolases such as caspases, since they are also functional at cytosolic $\mathrm{pH} .{ }^{23}$ In order to understand the role of the release of cathepsins $\mathrm{B}, \mathrm{D}$ and $\mathrm{L}$ in the cell death induced by $\mathrm{NH}_{2}-\mathrm{PS}$ NPs, pepstatin A (Pep A) and E64d were used to inhibit cathepsin D and cathepsins $\mathrm{B}$ and $\mathrm{L}$, respectively, prior to the treatment with NPs. ${ }^{33}$ ATP content was measured after $24 \mathrm{~h}$ of exposure to the NPs with or without the inhibitors. As shown in Fig. 3, the ATP levels of cells treated with the NPs were higher in the presence of E64d, but not in the presence of Pep A, which indicated that inhibition of cathepsin $\mathrm{B}$ and $\mathrm{L}$, but not $\mathrm{D}$, could protect cells from the damage induced by $\mathrm{NH}_{2}$-PS NPs. The release of cathepsins into the cytosol activates caspases and induces apoptosis in many cell death scenarios. ${ }^{23}$ When cathepsins B and L were inhibited (Fig. 3B), a decrease in caspase 3/7 activation was observed. However, the inhibition of cathepsins D with Pep A did not seem to affect the activation of caspase $3 / 7$. This suggested that cathepsin B and L, but not $\mathrm{D}$ might play a major role in the apoptotic cell death induced by these NPs. This is consistent with literature, where cathepsins $\mathrm{B}$ and $\mathrm{L}$, but not $\mathrm{D}$, have been shown to induce caspase-dependent or caspase-independent cell death. ${ }^{34}$

Additional measurements of ROS and cytosolic calcium levels in cells exposed to the NPs in the presence of cathepsin inhibitors (Fig. S9A and $\mathrm{B} \dagger$ ) also showed a decrease of their content when cathepsins were inhibited, thus further confirming that these signals can be related to the lysosomal damage induced by the NPs.

\subsection{Summary of cell death mechanisms}

By analyzing the signals activated by the NPs in a time resolved way, we propose a detailed description of the evolution of cell death in which lysosomal damage plays a central role (Fig. 4). At least two sub-populations of cells (colored as blue and red in Fig. 1 and $\mathrm{S} 1 \dagger$ ) at different stages of cell death could be distinguished.

Following nanoparticle uptake and accumulation in the lysosomes, at increasing exposure times the main cell population (blue) shows an increase in volume of acidic compartments and enlarged lysosomes, together with increased levels of vacuolization. At this time there is no alteration of mitochondrial membrane potential and the plasma membrane is still intact. After 3-6h of exposure, increased ROS levels are observed together with accumulation of autophagosomes, as suggested by the increase of LC3 II levels and the increased volume of acidic compartments in general. This could be indicative of activation of autophagy or impairment of the autophagic flux, although further studies are required to fully elucidate this.

The increase in the volume of acidic compartments (lysosomal enlargement) is also an upstream pro-apoptotic event, which has been observed in other cell death mechanisms induced by lysosomotropic agents. ${ }^{13}$ Enlarged lysosomes are known to be more susceptible to lysosomal damage. ${ }^{35}$ Here, at increasing exposure time, after the observed lysosomal damage upon NP accumulation, and sustained by ROS generation, the main cell population (blue) evolves into a population of strongly damaged and dying cells (red). Cells in this sub-population show loss of LysoTracker Red intensity, together with other cellular features such as shrinkage of cell size (decreased FS) 


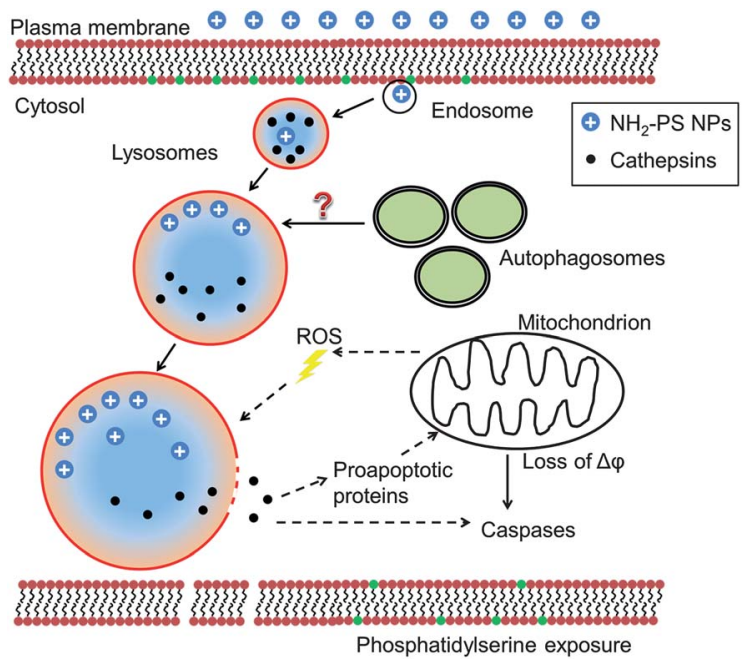

Fig. 4 Mechanism of cell death induced by $\mathrm{NH}_{2}-$ PS NPs. NPs enter the cells and within $1 \mathrm{~h}$ of exposure are trafficked to lysosomes. After 3-6 h exposure, accumulation of the NPs into lysosomes leads to their enlargement. After 6-8 $\mathrm{h}$ exposure, release of lysosomal proteases (such as cathepsins B, D and L) into cytosol is observed, which can activate downstream apoptotic executors causing damage to mitochondria (loss of mitochondrial membrane potential), and other apoptotic events (caspase 3/7 activation, phosphatidylserine exposure). Finally plasma membrane permeabilization and cell death occur. ROS production plays an important role by amplifying cell death and formation of a positive feedback loop between mitochondria and lysosomes.

and increasing granularity (SS), loss of mitochondrial membrane potential and of plasma membrane integrity. These are cells at the late stage of cell death. Release of lysosomal contents, such as cathepsins B, D and L into the cytosol, leads to damage to the mitochondria. Once mitochondria are impaired, which marks the point of no return for cell death, caspase 3/7 activation and exposure of phosphatidylserine are observed, followed by loss of cell membrane integrity and ultimately cell death.

A small percentage of cells in this population has been observed as soon as $1 \mathrm{~h}$ after exposure to $\mathrm{NH}_{2}$-PS NPs, perhaps explained by varying levels of uptake and different sensitivity of individual cells within the population.

The results obtained with the use of inhibitors further confirm the central role of the release of lysosomal content in the observed cell death and seems to suggest that the presence of second messengers such as ROS and cytosolic calcium may also be connected to the damage to the lysosomes.

\section{Conclusions}

In order to understand the mechanisms of cell death induced by

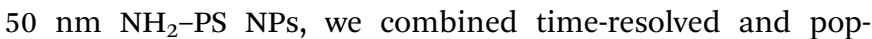
ulation-discriminated flow cytometry analysis, confocal imaging and other biomolecular approaches. Overall, the study of the interplay between the cell death mechanisms clearly suggests that most of the events detected in cells exposed to the $\mathrm{NH}_{2}-\mathrm{PS}$ NPs can be explained with the strong impact that these NPs have on lysosomes.
The details of the mechanism by which these positively charged particles induce such damage to the lysosomes still remain unresolved: clearly the initial presence of the corona on the nanoparticle surfaces and its progressive degradation at lysosomal level play a crucial role, ${ }^{9}$ however more studies will be required to fully address this point.

More in general, since several NPs have been shown to accumulate inside the lysosomes and often with no sign of export or degradation, ${ }^{36-38}$ it will be important to assess potential NP effects on lysosomal functions, even when there is no lysosomal impact as evident as what described here for these $\mathrm{NH}_{2}-\mathrm{PS}$ NPs.

\section{Acknowledgements}

This work was conducted under the framework of the INSPIRE programme, funded by the Irish Government's Programme for Research in Third Level Institutions, Cycle 4, National Development Plan 2007-2013, and is based upon work supported by Science Foundation Ireland (SFI) under Grant no. (SFI/SRC/ B1155), the EU FP7 Capacities project QualityNano (EU 262163), and the EU FP7 Small Collaborative Project NeuroNano (NMP4SL-2008-214547). The JCS lab is supported by a Principal Investigator grant (09/IN.1/B2604) from SFI. Flow Cytometry and Confocal Microscopy have been performed using the UCD Conway Institute Core Facilities.

\section{Notes and references}

1 Y. Zhao, S. Zhang, S. Cui, B. Wang and S. Zhang, Expert Opin. Drug Delivery, 2011, 9, 127-139.

2 X. Sun, C. Liu, D. Liu, P. Li and N. Zhang, Int. J. Pharm., 2012, 425, 62-72.

3 A. Akinc, M. Thomas, A. M. Klibanov and R. Langer, J. Gene Med., 2005, 7, 657-663.

4 T. Xia, M. Kovochich, M. Liong, J. I. Zink and A. E. Nel, ACS Nano, 2008, 2, 85-96.

5 S. Hong, P. R. Leroueil, E. K. Janus, J. L. Peters, M. M. Kober, M. T. Islam, B. G. Orr, J. R. Baker and M. M. Banaszak Holl, Bioconjugate Chem., 2006, 17, 728-734.

6 P. Ruenraroengsak, P. Novak, D. Berhanu, A. J. Thorley, E. Valsami-Jones, J. Gorelik, Y. E. Korchev and T. D. Tetley, Nanotoxicology, 2012, 6, 94-108.

7 K. A. Dawson, A. Salvati and I. Lynch, Nat. Nanotechnol., 2009, 4, 84-85.

8 M. P. Monopoli, C. Åberg, A. Salvati and K. A. Dawson, Nat. Nanotechnol., 2012, 7, 779-786.

9 F. Wang, L. Yu, M. P. Monopoli, P. Sandin, E. Mahon, A. Salvati and K. A. Dawson, Nanomedicine: N. B. M., 2013, in press, http://dx.doi.org/10.1016/j.nano.2013.04.010.

10 M. G. Bexiga, J. A. Varela, F. Wang, F. Fenaroli, A. Salvati, I. Lynch, J. C. Simpson and K. A. Dawson, Nanotoxicology, 2011, 5, 557-567.

11 C. Settembre, A. Fraldi, L. Jahreiss, C. Spampanato, C. Venturi, D. Medina, R. de Pablo, C. Tacchetti, D. Rubinsztein and A. Ballabio, Hum. Mol. Genet., 2008, 17, 119-129. 
12 A. Eisenberg-Lerner, S. Bialik, H. U. Simon and A. Kimchi, Cell Death Differ., 2009, 16, 966-975.

13 E. Agostinelli and N. Seiler, Int. J. Oncol., 2007, 31, 473-484. 14 P. Boya, R. González-Polo, N. Casares, J. Perfettini, P. Dessen,

N. Larochette, D. Métivier, D. Meley, S. Souquere, T. Yoshimori, G. Pierron, P. Codogno and G. Kroemer, Mol. Cell. Biol., 2005, 25, 1025-1040.

15 Y. H. Yoon, K. S. Cho, J. J. Hwang, S.-J. Lee, J. A. Choi and J.-Y. Koh, Invest. Ophthalmol. Visual Sci., 2010, 51, 60306037.

16 T. Thomas, I. Majoros, A. Kotlyar, D. Mullen, M. Holl and J. Baker, Biomacromolecules, 2009, 10, 3207-3214.

17 M. E. Guicciardi, M. Leist and G. J. Gores, Oncogene, 2004, 23, 2881-2890.

18 C. Oberle, J. Huai, T. Reinheckel, M. Tacke, M. Rassner, P. G. Ekert, J. Buellesbach and C. Borner, Cell Death Differ., 2010, 17, 1167-1178.

19 K. Ono, S. O. Kim and J. Han, Mol. Cell. Biol., 2003, 23, 665676.

20 K. Unfried, C. Albrecht, L.-O. Klotz, A. Von Mikecz, S. Grether-Beck and R. Schins, Nanotoxicology, 2007, 1, 5271.

21 G. Kroemer, B. Dallaporta and M. Resche-Rigon, Annu. Rev. Physiol., 1998, 60, 619-642.

22 D. R. Green and J. C. Reed, Science, 1998, 281, 1309-1312.

23 P. Boya and G. Kroemer, Oncogene, 2008, 27, 6434-6451.

24 V. Stoka, B. Turk, S. L. Schendel, T.-H. Kim, T. Cirman, S. J. Snipas, L. M. Ellerby, D. Bredesen, H. Freeze, M. Abrahamson, D. Brömme, S. Krajewski, J. C. Reed,
X.-M. Yin, V. Turk and G. S. Salvesen, J. Biol. Chem., 2001, 276, 3149-3157.

25 S. Stern, P. Adiseshaiah and R. Crist, Part. Fibre Toxicol., 2012, 9, 20.

26 D. Klionsky, Nat. Rev. Mol. Cell Biol., 2007, 8, 931-937.

27 N. Mizushima and T. Yoshimori, Autophagy, 2007, 3, 542545.

28 I. Tanida, N. Minematsu-Ikeguchi, T. Ueno and E. Kominami, Autophagy, 2005, 1, 84-91.

29 X. Ma, Y. Wu, S. Jin, Y. Tian, X. Zhang, Y. Zhao, L. Yu and X.-J. Liang, ACS Nano, 2011, 5, 8629-8639.

30 G. Kroemer and S. J. Martin, Nat. Med., 2005, 11, 725-730.

31 M. C. Maiuri, E. Zalckvar, A. Kimchi and G. Kroemer, Nat. Rev. Mol. Cell Biol., 2007, 8, 741-752.

32 B. Turk, V. Stoka, J. Rozman-Pungercar, T. Cirman, G. DrogaMazovec, K. Oreic and V. Turk, Biol. Chem., 2002, 383, 10351044.

33 C. E. Chwieralski, T. Welte and F. Bühling, Apoptosis, 2006, $11,7$.

34 R. Ishisaka, K. Utsumi and T. Utsumi, Biosci., Biotechnol., Biochem., 2002, 66, 1865-1872.

35 G. Kroemer and M. Jaattela, Nat. Rev. Cancer, 2005, 5, 886897.

36 J. A. Kim, C. Åberg, A. Salvati and K. A. Dawson, Nat. Nanotechnol., 2012, 7, 62-68.

37 A. Salvati, C. Åberg, T. dos Santos, J. Varela, P. Pinto, I. Lynch and K. A. Dawson, Nanomedicine, 2011, 7, 818-826.

38 K. Shapero, F. Fenaroli, I. Lynch, D. C. Cottell, A. Salvati and K. A. Dawson, Mol. Biosyst., 2010, 7, 371-378. 